\title{
ARTICLE
}

Received 19 Oct 2013 | Accepted 25 Feb 2014 | Published 24 Mar $2014 \quad$ DOl: 10.1038/ncomms4504

\section{Plant GSK3 proteins regulate xylem cell differentiation downstream of TDIF-TDR signalling}

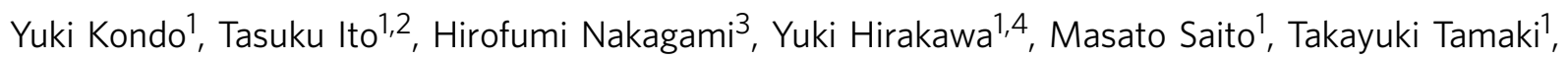
Ken Shirasu ${ }^{3} \&$ Hiroo Fukuda ${ }^{1}$

During plant radial growth typically seen in trees, procambial and cambial cells act as meristematic cells in the vascular system to self-proliferate and differentiate into xylem cells. These two processes are regulated by a signalling pathway composed of a peptide ligand and its receptor; tracheary element differentiation inhibitory factor (TDIF) and TDIF RECEPTOR (TDR). Here we show that glycogen synthase kinase 3 proteins (GSK3s) are crucial downstream components of the TDIF signalling pathway suppressing xylem differentiation from procambial cells. TDR interacts with GSK3s at the plasma membrane and activates GSK3s in a TDIF-dependent fashion. Consistently, a specific inhibitor of plant GSK3s strongly induces xylem cell differentiation through BRI1-EMS SUPPRESSOR 1 (BES1), a well-known target transcription factor of GSK3s. Our findings provide insight into the regulation of cell fate determination in meristem maintenance.

\footnotetext{
${ }^{1}$ Department of Biological Sciences, Graduate School of Science, The University of Tokyo, 7-3-1 Hongo, Bunkyo-ku, Tokyo 113-0033, Japan. ${ }^{2}$ Department of Integrated Genetics, National Institute of Genetics, Yata 1111, Mishima, Shizuoka 411-8540, Japan. ${ }^{3}$ RIKEN Center for Sustainable Resource Science, 7-22 Suehiro-cho, Tsurumi-ku, Kanagawa, Yokohama, Japan. ${ }^{4}$ School of Biological Sciences, Monash University, Melbourne, Victoria 3800, Australia. Correspondence and requests for materials should be addressed to Y.K. (email: p@biol.s.u-tokyo.ac.jp) or to H.F. (email: fukuda@biol.s.u-tokyo.ac.jp).
} 
$\mathrm{P}$ lants keep growing throughout their lives owing to the activity of stem cells in meristems ${ }^{1}$. Genetic analyses have revealed key players such as ligands, receptors and transcription factors involved in plant meristem maintenance ${ }^{1,2}$. The CLAVATA3 (CLV3)/EMBRYO SURROUNDING REGIONrelated (CLE) family of signalling peptides plays crucial roles in the regulation of stem cell maintenance in the meristems ${ }^{3}$. In the shoot apical meristem of Arabidopsis thaliana, CLV3 peptide secreted from stem cells restricts the expression domain of the WUSCHEL (WUS) homeobox transcription factor gene to the organizing centre via three receptor pathways, CLV1, CLV2CORYNE (CRN) and RECEPTOR-LIKE PROTEIN KINASE 2 $(\mathrm{RPK} 2)^{4-10}$. CLV3-WUS signalling makes a negative feedback loop to maintain the homoeostasis of stem cell population in the shoot apical meristem ${ }^{11-13}$. A similar CLE peptide-receptortranscription factor system is involved in the maintenance of columella stem cells in the root apical meristem ${ }^{14}$. Previous studies have revealed the importance of individual peptide signals in meristem maintenance, however, the manner of intracellular signal transduction mechanisms regulating cell fates is still a mystery.

The CLE peptide, tracheary element differentiation inhibitory factor (TDIF), which was isolated as a dodecapeptide with two hydroxyproline residues from a Zinnia elegans xylogenic culture, plays a central role in the maintenance of procambial cells ${ }^{15}$. In Arabidopsis, TDIF is encoded by two CLE genes, CLE41 and CLE44 (refs 15,16). Genetic and biochemical analyses identified TDIF receptor (TDR)/PHLOEM INTERCALATED WITH XYLEM (PXY), which belongs to the leucine-rich repeat receptor-like kinase (LRR-RLK) class XI, as a specific membrane-associated receptor for TDIF $^{16,17}$. TDIF both suppresses the differentiation of procambial cells into xylem cells and promotes their proliferation through $\mathrm{TDR}^{15,16}$. These dual functions of TDIF-TDR are considered to be separated by downstream components ${ }^{18}$. A transcription factor WUSCHEL-related HOMEOBOX 4 (WOX4) is a target of the TDIF-TDR signalling pathway leading to cell proliferation but not to xylem differentiation ${ }^{18}$. However, until now, the intracellular signalling components leading to the inhibition of xylem differentiation remain elusive.

In this study, we show the identification of BRASSINOSTEROID-INSENSITIVE 2 (BIN2), a member of plant GSK3s, as an interacting protein against TDR cytoplasmic kinase domain by yeast two-hybrid $(\mathrm{Y} 2 \mathrm{H})$ screening. We also show that SKI and II members in the GSK3s family interact with TDR at the plasma membrane (PM) in plant cells, and their interactions are regulated in a TDIF-dependent fashion. Our genetic and anatomical analyses suggest that GSK3s redundantly mediate TDIF-TDR signalling in the aspect of xylem differentiation, but not of procambial cell proliferation. We finally show the identification of a transcription factor, BES1 that acts downstream of TDIF-TDR-GSK3s pathway. This TDIF-TDR-GSK3s-BES1 pathway is genetically independent of the TDIF-TDR-WOX4 pathway in the maintenance of procambial cells. Our results establish a model whereby the TDIF-TDR activates GSK3s, which in turn suppresses BES1 that promotes xylem differentiation from procambial cells.

\section{Results}

BIN2 interacts with TDR cytoplasmic domain in Y2H assay. To investigate the signalling pathway downstream of TDR, we carried out $\mathrm{Y} 2 \mathrm{H}$ screen using the cytoplasmic kinase domain of TDR (TDRKD). BIN2, a member of GSK3s-mediating intracellular BR signalling ${ }^{19}$, was identified as a potential interacting factor from approximately 5,000,000 clones (Fig. 1a). Detailed $\mathrm{Y} 2 \mathrm{H}$ assays showed that the full length BIN2 protein interacts with TDRKD, but not with the kinase domain of CRN, another receptor-like protein functioning in CLE signalling ${ }^{9,20,21}$ (Fig. 1a, and Supplementary Fig. 1). In Arabidopsis, there are 10 members of GSK3s, consisting of 4 subgroups SKI-IV 22 (Fig. 1b,c). Comprehensive $\mathrm{Y} 2 \mathrm{H}$ analyses revealed that TDRKD interacts with all members of the SKII (BIN2, BIN2-LIKE 1 (BIL1) and BIL2) subgroup (Fig. 1b). In addition, TDRKD showed a strong interaction with ATSK11-ATSK13 and a weak interaction with ATSK32 and ATSK41 (Fig. 1b). A pull-down assay with purified TDRKD and either BIN2 or BIL1 confirmed a physical interaction between TDRKD and both of the two plant GSK3s (Supplementary Fig. 2).

TDR interacts with BIN2 at the PM in plant cells. Next, we reconstructed the TDIF-TDR-BIN2 pathway in Nicotiana benthamiana leaves, with constructs of TDR fused with the cyan fluorescent protein (TDR-CFP) under the control of an estradiolinducible system and BIN2 fused with the yellow fluorescent protein (BIN2-YFP) directed by the CaMV 35S promoter (Fig. 2a-c). BIN2-YFP localized to the PM, cytoplasm and nucleus, consistently with the previous report ${ }^{23}$ (Supplementary Fig. 3). TDR-CFP localized specifically to the PM at 12 and $24 \mathrm{~h}$ after estradiol induction (Supplementary Fig. 4). Fluorescence resonance energy transfer (FRET) analysis ${ }^{24,25}$ revealed higher FRET efficiency of TDR-CFP with BIN2-YFP than that of RPK2-CFP (Fig. 2d), which acts as a receptor in CLV3 signalling ${ }^{10}$. This FRET analysis indicates a close physical association between TDR and BIN2 at the PM in plant cells. Overexpression of CLE41, which encodes TDIF, but not of CLV3 reduced greatly the FRET efficiency between TDR-CFP and BIN2-YFP (Fig. 2d). Similarly, TDIF but not CLV3 peptide treatment reduced the FRET efficiency within $30 \mathrm{~min}$ (Fig. 2e). These decreases in the FRET efficiency were lost by a mutation in the ATP-binding domain of TDR (TDRK747E-CFP) (Fig. 2d,e), which is expected to extinguish the kinase activity ${ }^{26}$. These results indicate that FRET efficiency between TDR and BIN2 is decreased upon TDIF through the TDR kinase activity, which suggests the possibilities of BIN2 dissociation from TDR or conformational changes in TDR-BIN2.

TDIF promotes BIN2 activity through TDR in procambial cells. BIN2 kinase activity can be measured by BRASSINAZOLE RESISTANT 1 (BZR1)-CFP movement from the nucleus to cytoplasm as a sensor ${ }^{27-30}$. Overexpression of BIN2 reduced nuclear localization of BZR1-CFP in N. benthamiana epidermis and this reduction was overcome by suppression of BIN2 activity with bikinin, a plant GSK3 specific inhibitor (Fig. 3a-e). To investigate BIN2 action upon TDIF treatment in procambial cells of Arabidopsis, BZR1-GFP expression was driven under the TDR promoter in the wild type (WT) and $t d r-1$ (Supplementary Fig. 5a,b). In the WT, the level of BZR1-GFP nuclear localization was decreased within $60 \mathrm{~min}$ upon TDIF treatment, while this decrease was not observed in $t d r-1$ (Fig. $3 \mathrm{f}-\mathrm{m}$ and Supplementary Fig. $5 c, d)$. Bikinin suppressed this TDIF-dependent decrease in the WT (Fig. $3 \mathrm{f}-\mathrm{m})$. All of these data strongly suggest that TDIF positively regulates BIN2 kinase activity through endogenous TDR in procambial cells.

SKI and II members redundantly mediate TDIF signalling. Next, we examined the involvement of other GSK3s than BIN2 in TDIF signalling. In FRET analysis, TDR showed high FRET efficiency with BIL1, BIL2, ATSK11 and ATSK13, but low efficiency with ATSK12, ATSK31, ATSK32 and ATSK41 (Supplementary Fig. 6). Upon TDIF treatment, TDR exhibited reduction in FRET efficiency strongly with BIL2 and ATSK13, slightly with ATSK11 and hardly with BIL1 (Supplementary 
a
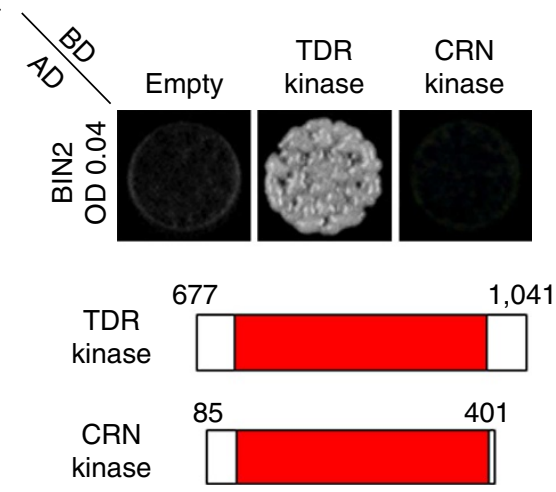

C

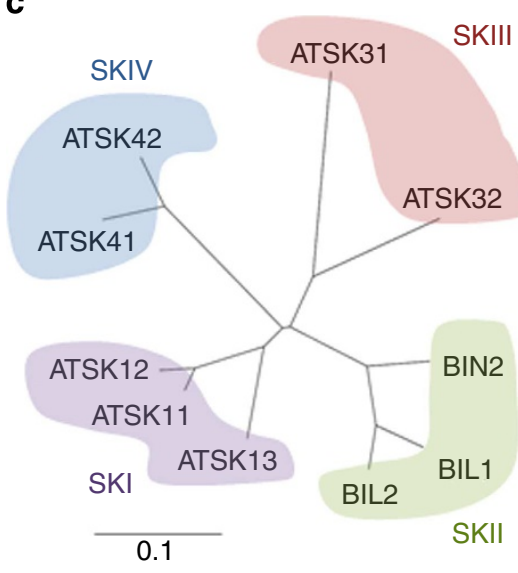

b

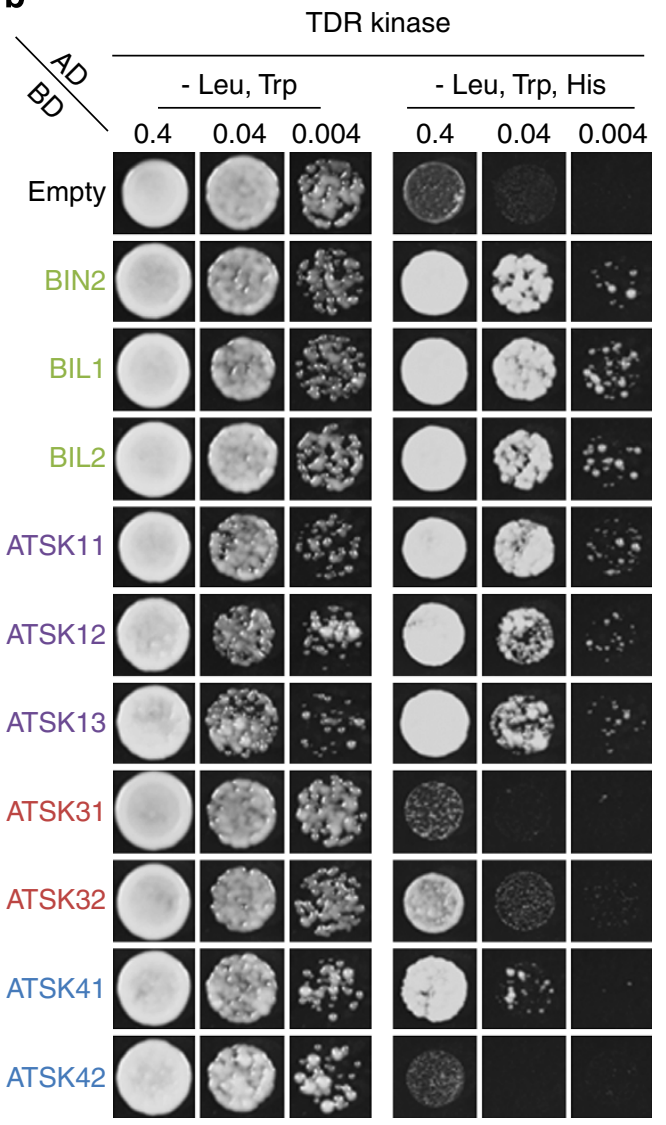

Figure 1 | Interaction between TDR and GSK3s in yeast. (a,b) TDR interacts with GSK3s in Y2H analysis. (a) Top panel: cultured yeasts harbouring both pACTII and pGBKT7 vectors were spread on SD/-Leu/-Trp/-His plates. Bottom panel: schematic diagram of TDR and CRN intracellular domain used in this study. The predicted kinase domains are shown in red. (b) Cultured yeasts were spread on SD/-Leu/-Trp and SD/-Leu/-Trp/-His plates at various concentrations. (c) A molecular phylogenetic tree for GSK3s of Arabidopsis. The phylogenetic tree was constructed on the basis of neighbour-joining method.
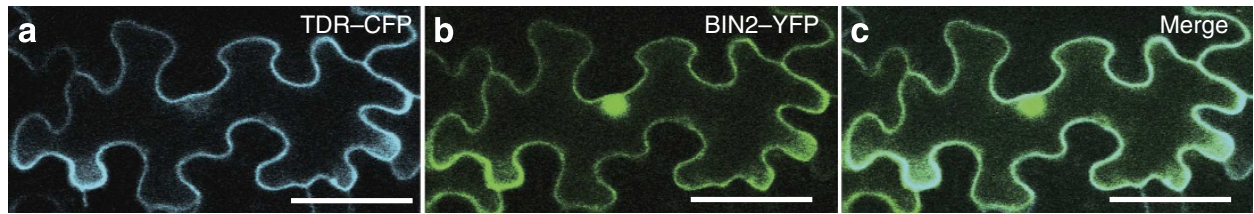

d

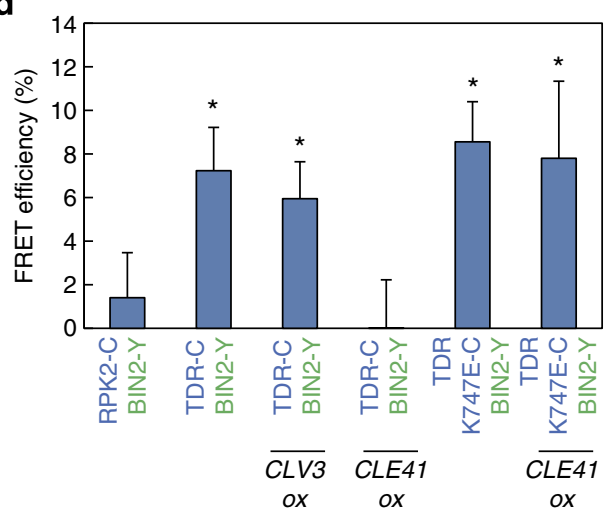

e

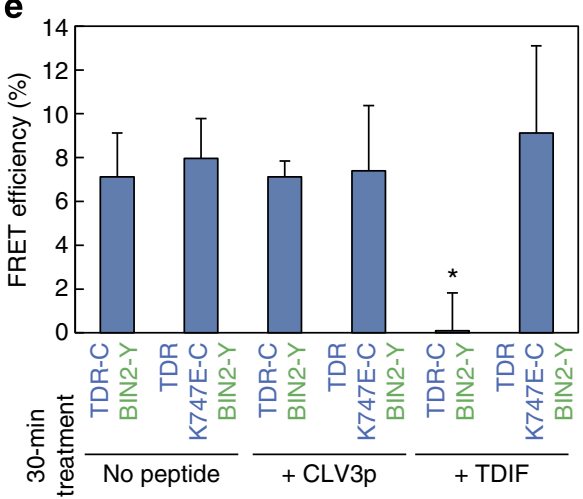

Figure 2 | Interaction between TDR and BIN2 in plant cells. (a-c) TDR-CFP and BIN2-YFP expressed simultaneously for FRET analysis in N. benthamiana. (d,e) The effect of CLE genes and peptides on FRET efficiencies between CFP and YFP fused with various proteins. (d) CLV3 and CLE41 (encoding TDIF) were simultaneously expressed using the estradiol-inducible system $(n \geq 15)$. (e) Chemically synthesized CLV3 peptide and TDIF were applied for $30 \mathrm{~min}$ at $5 \mu \mathrm{M}$ concentration $(n \geq 10)$. Asterisks indicate significant differences according to the Student's $t$-test $(P<0.001)$. Error bars indicate s.d. 

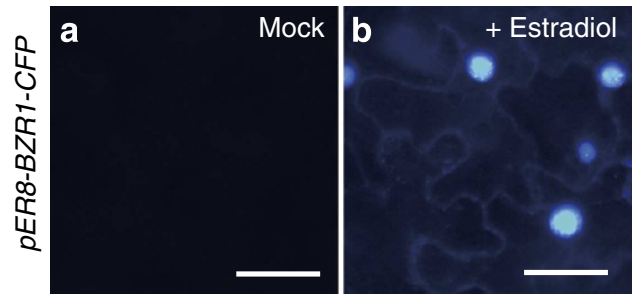

$35 S-B I N 2$

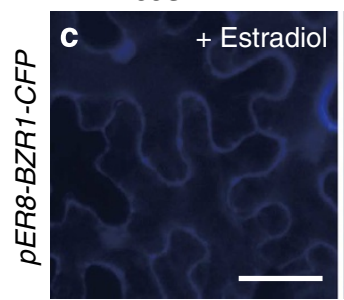

$35 S-B I N 2$
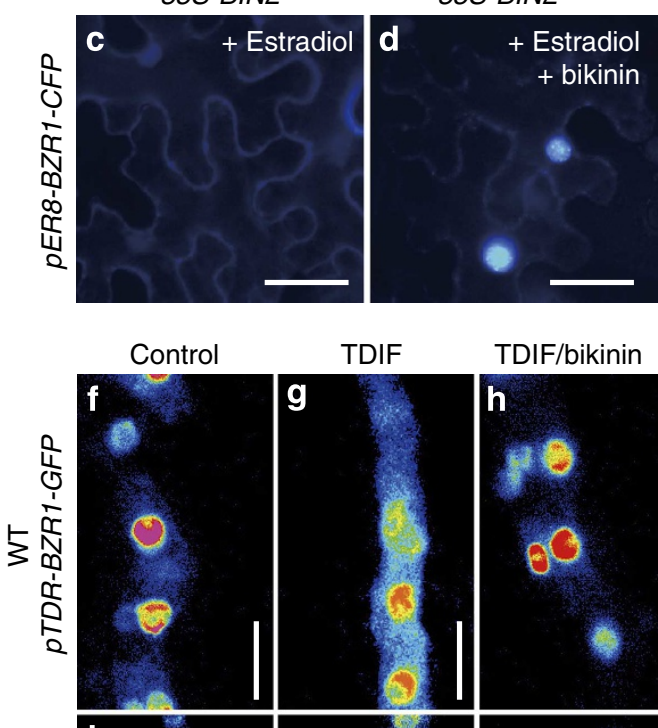

TDIF

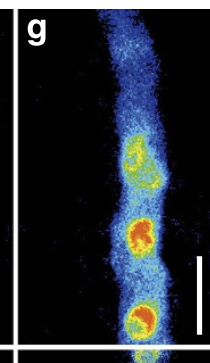

TDIF/bikinin
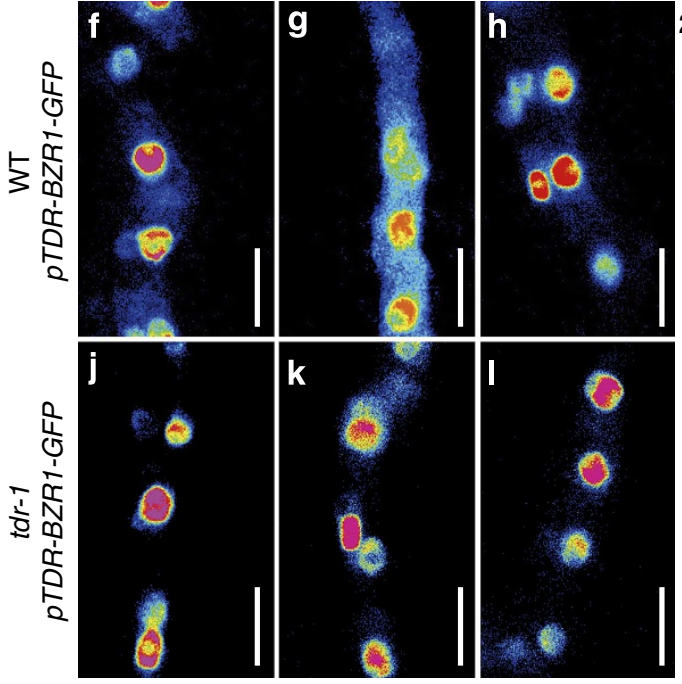

e

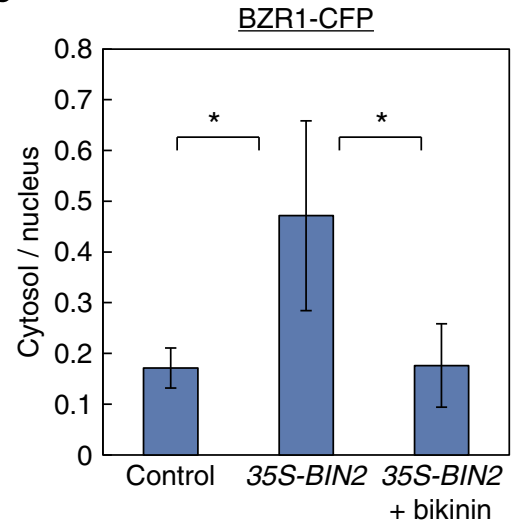

i

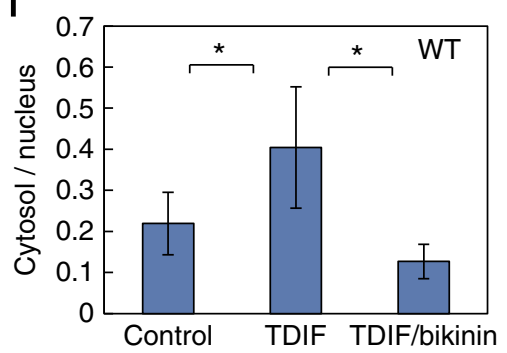

m

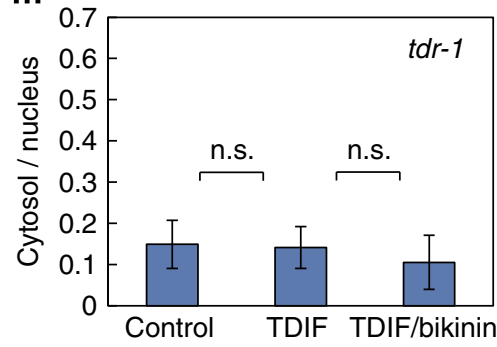

Figure 3 | TDIF-dependent activation of BIN2 in procambial cells. (a-d) Effect of BIN2 activity on BZR1-CFP localization in N. benthamiana. BZR1-CFP was induced with $5 \mu \mathrm{M}$ estradiol treatment for $6 \mathrm{~h}(\mathbf{b}-\mathbf{d}$ ). (a) mock (DMSO) treatment. (b) estradiol treatment. (c) estradiol treatment and BIN2 co-expression (d) estradiol treatment, BIN2 co-expression and an additional $2 \mathrm{~h}$ of $10 \mu \mathrm{M}$ bikinin treatment. (e) The cytosol-to-nucleus ratios of the fluorescent signal intensity of BZR1-CFP as influenced by the co-expression of BIN2 and the treatment of bikinin were shown ( $n \geq 25$ ). (f-m) Measurement of BIN2 activity in Arabidopsis procambial cells. Fluorescent intensities of 5-day-old WT and tdr-1 seedlings harbouring PTDR-BZRT-GFP were shown as colour-coded images, which relate to the scale. Leaves were treated with mock (f,j) or with $5 \mu \mathrm{M}$ TDIF $(\mathbf{g}, \mathbf{k})$, or with both TDIF and $10 \mu \mathrm{M}$ bikinin $(\mathbf{h}, \mathbf{l})$ for $60 \mathrm{~min}$. (i,m) The cytosol-to-nucleus ratios of the fluorescent signal intensity were calculated $(\mathrm{n} \geq 25$ ) for WT (i) and $t d r-1(\mathbf{m})$. Asterisks indicate significant differences according to the Student's $t$-test $(P<0.001)$. n.s. indicates no significant difference according to the Student's t-test. Error bars indicate s.d. Scale bars, $100 \mu \mathrm{m}$ for (a-d) and $50 \mu \mathrm{m}$ for (f-I). DMSO, dimethyl sulfoxide.

Fig. 6). Together with $\mathrm{Y} 2 \mathrm{H}$ results, these results suggest an involvement of SKI and II subgroup members in TDIF signalling (Supplementary Table 1). To investigate roles of SKI and II members in TDIF signalling, TDIF sensitivity was examined by genetic analysis using loss-of-function mutants for GSK3s. Previous physiological and genetic studies have shown that TDIF has two distinct effects on vascular development; inhibition of xylem differentiation and promotion of vascular cell division ${ }^{15,16,18}$. In the WT, exogenous TDIF application suppresses xylem differentiation from procambial cells, resulting in discontinuous xylem formation in Arabidopsis leaf veins ${ }^{16,18}$ (Fig. 4a,b). bin2-3 mutant showed a weak resistance to TDIF in terms of xylem differentiation (Fig. 4c,d,i). bil2 enhanced TDIF resistance of $\operatorname{bin} 2-3$ at $100 \mathrm{nM}$ concentration $(P<0.01$ by Student's $t$-test), while bil1 did not $(P=0.07$ by Student's $t$-test $)$ (Fig. 4i), indicating a differential role in TDIF signalling between
BIL1 and BIN2/BIL2. Consistent with genetic results, expression of pBIN2-GUS and pBIL2-GUS but not of pBIL1-GUS was observed along the leaf vein, overlapping with TDR expression domain (Fig. 5a-h). Although bin2-3billbil2 (bin2 triple) mutant showed as strong resistance as bin2-3bil2 double mutant $(P=0.15$ by Student's $t$-test; at $100 \mathrm{nM})(P=0.11$ by Student's $t$-test; at $1,000 \mathrm{nM}$ ), introduction of interfering RNA (RNAi) against ATSK13 in the bin2 triple mutant background (hereafter called gsk3 quadruple mutant), which resulted in about 50\% knockdown of ATSK13 (Supplementary Fig. 7a), severely destroyed the TDIF sensitivity $(P<0.01$ by Student's $t$-test; at both concentrations) (Fig. $4 \mathrm{e}-\mathrm{i}$ ). These results indicate that GSK3s redundantly mediate TDIF signalling in terms of regulating xylem differentiation. Next, to evaluate the effect of TDIF on vascular cell proliferation, vascular cell number in hypocotyls was counted with the distinction of four different cell types; xylem, 

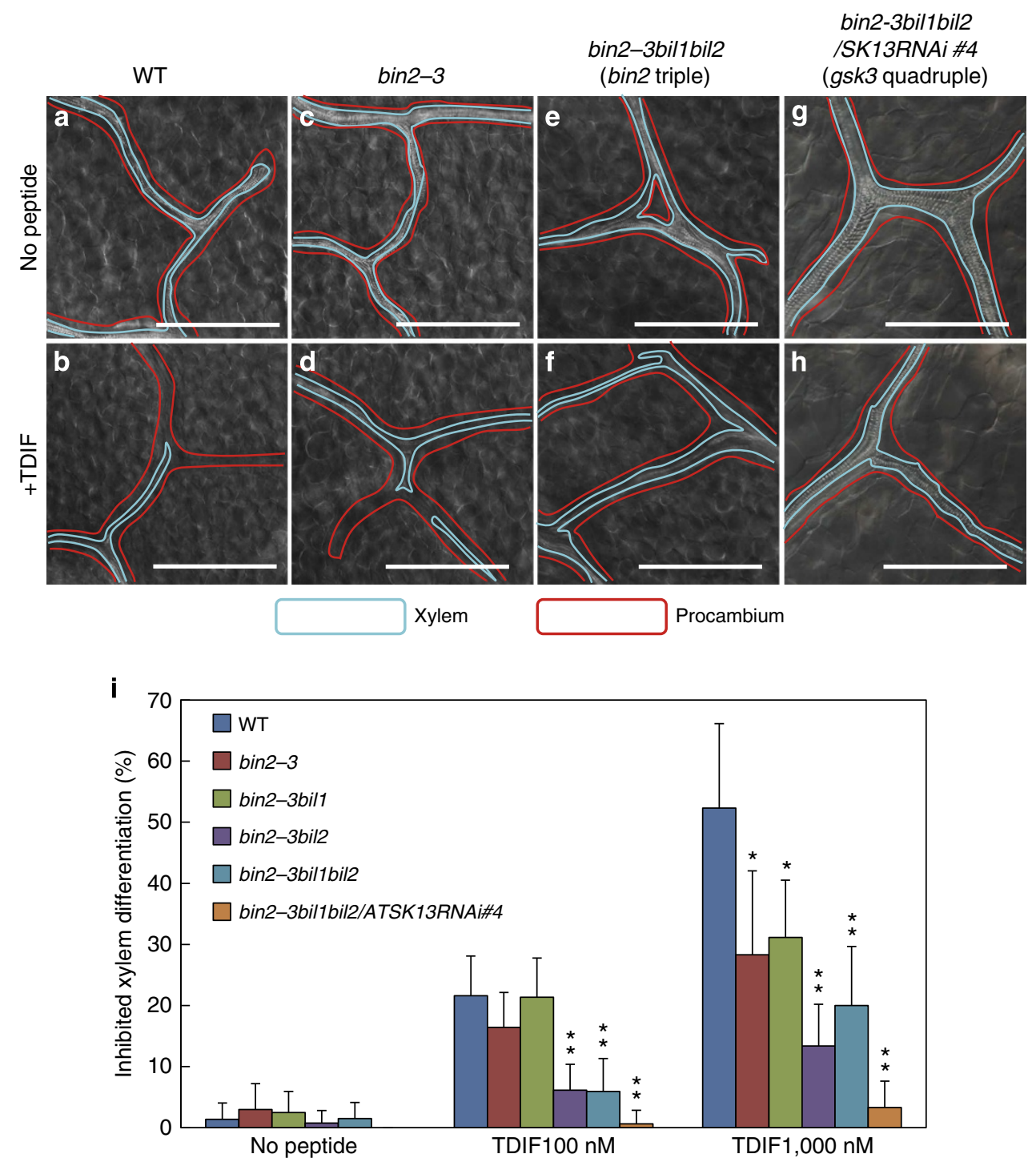

Figure 4 | TDIF sensitivity of gsk3 mutants in terms of xylem differentiation. (a-h) Arabidopsis seeds for Ws (WT), bin2-3, bin2 triple and gsk3 quadruple mutants were germinated in liquid MS medium with or without $1000 \mathrm{nM}$ TDIF. The vasculature of true leaves from 11-day-old seedlings was shown. Blue and red lines indicate the areas of xylem and procambial cells, respectively. Scale bar, $200 \mu \mathrm{m}$. (i) The effect of TDIF on xylem differentiation in WT and gsk3 mutants was calculated by the frequencies of inhibited xylem differentiation in high order veins $(n \geq 8)$. Error bars indicate SD. Asterisks indicate significant differences using the Student's $t$-test $\left({ }^{\star} P<0.01,{ }^{\star \star} P<0.001\right)$.

procambium, phloem and uncharacterized cells (Supplementary Fig. 8a). Here we termed procambial cells as undifferentiated cells between xylem and phloem cells, and uncharacterized cells as undifferentiated vascular cells and divided pericycle cells located outside the original xylem cells (Supplementary Fig. 8a). In the WT, TDIF application increased the number of procambial cells and uncharacterized cells. Similarly to the WT, the gsk3 quadruple mutant showed TDIF-dependent increment in the number of procambial and uncharacterized cells (Fig. 6), suggesting that GSK3s are not likely to contribute to TDIFdependent promotion of vascular cell proliferation. In hypocotyls, TDIF did not reduce xylem cell numbers (Fig. 6e), which is consistent with results reported previously $16,31,32$. This might result from a counterbalance between TDIF-dependent reduced xylem cells and ectopic xylem formation as a secondary effect arising from TDIF-induced random procambial cell proliferation (Fig. 6b arrows). In the absence of TDIF, the gsk3 quadruple mutant showed the increment in the number of all types of vascular cells except for procambial cells, when compared with the WT $(P<0.05$ by Student's $t$-test) (Fig. 6e). To normalize the number of vascular cells, we calculated the ratio of each cell type in total vascular cell number. Although the gsk3 quadruple mutant significantly increased the xylem ratio $(P<0.05$ by Student's $t$-test) and decreased the procambium ratio $(P<0.005$ by Student's $t$-test), it did not change other ratios (Supplementary Fig. 8b). Considering that BIN2 and BIL2 were expressed broadly in vascular cells including procambial cells (Fig. 5i,j), these results indicate that GSK3s repress xylem differentiation from procambial cells in hypocotyls. All these data suggest that GSK3s mediate TDIF signalling in the regulation of xylem differentiation from procambial cells but not of procambial cell proliferation.

GSK3s contribute to the procambium maintenance together with WOX4. In the vascular development, defect in TDR leads to the reduction of procambial cells between xylem and phloem cells in hypocotyls ${ }^{17,18}$. In severer cases, it often $(<70 \%)$ induced the 

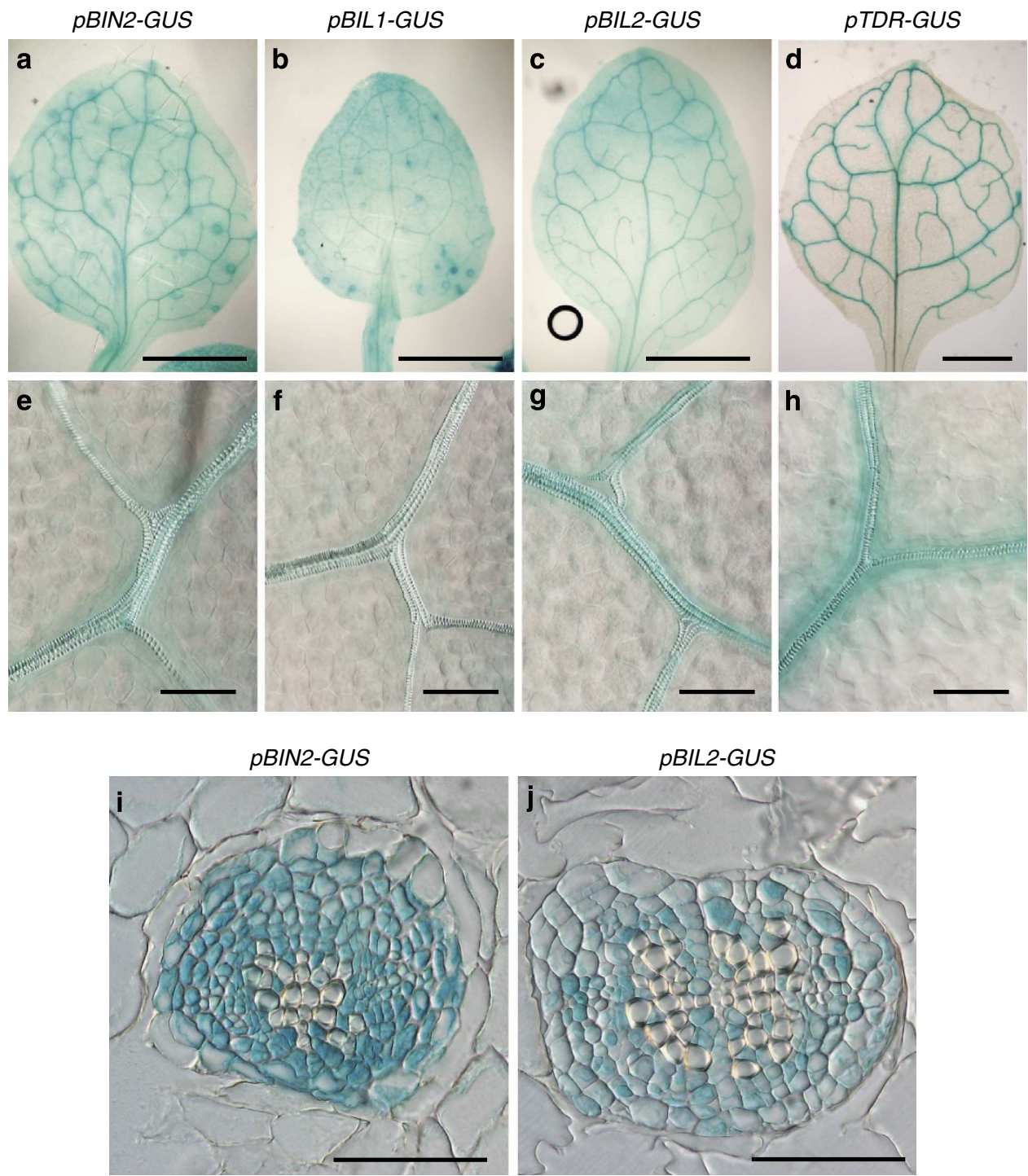

Figure 5 | Expression patterns of pBIN2-GUS, pBIL1-GUS, pBIL2-GUS, and pTDR-GUS. (a-h) GUS activity was examined in rosette leaves (a-d) and leaf veins (e-h) of transgenic Arabidopsis plants harbouring pBIN2-GUS, pBIL1-GUS, pBIL2-GUS and pTDR-GUS. (i,j) Transverse sections of hypocotyls in 10-day-old Arabidopsis seedlings harbouring pBIN2-GUS and pBIL2-GUS. Scale bars, $1 \mathrm{~mm}$ for (a-d) and $50 \mu \mathrm{m}$ for $(\mathbf{e}-\mathbf{j})$.
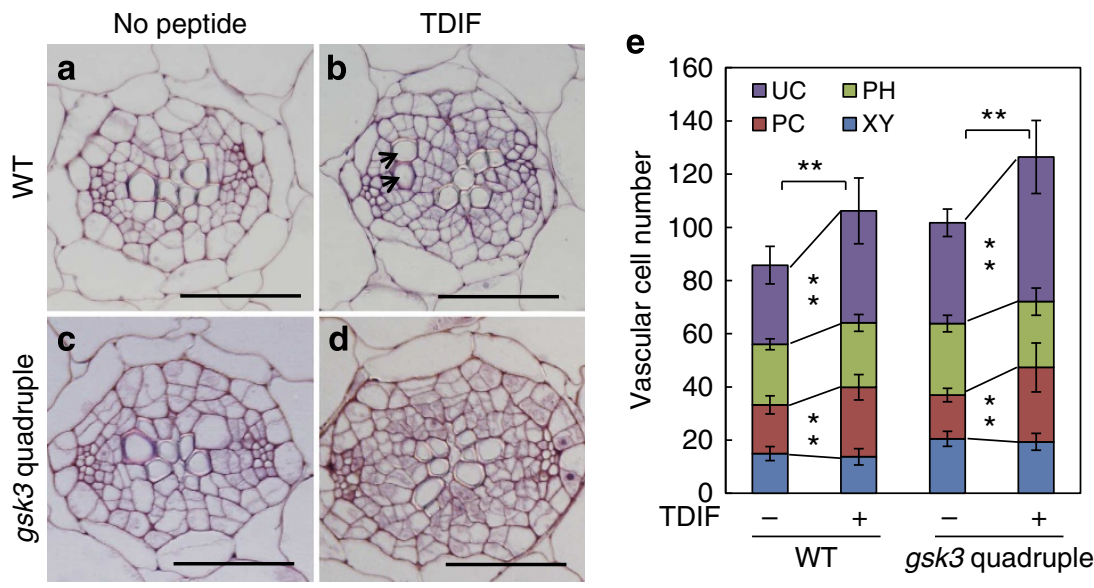

Figure 6 | TDIF sensitivity of $\mathbf{g s k 3}$ quadruple mutants in terms of vascular cell proliferation. (a-d) Cross sections of hypocotyls of Ws (a,b) and gsk3 quadruple mutants $(\mathbf{c}, \mathbf{d})$ grown for 7 days with $(\mathbf{b}, \mathbf{d})$ or without $1000 \mathrm{nM}$ TDIF $(\mathbf{a}, \mathbf{c})$. Scale bars, $50 \mu$ m. (e) Vascular cell number was calculated from images obtained from hypocotyl sections of 7-day-old WT and gsk3 quadruple mutants by classified into four different cell types (according to Supplementary Fig. 8a) $(n=12)$. Error bars indicate s.d. Asterisks indicate significant differences using the Student's $t$-test $\left({ }^{\star} P<0.01,{ }^{\star \star} P<0.001\right)$. $\mathrm{XY}, \mathrm{PH}, \mathrm{PC}$ and $\mathrm{UC}$ indicate that xylem, phloem, procambium and uncharacterized cells, respectively. 
formation of xylem cells adjacent to phloem cells in hypocotyls ${ }^{17,18}$ (Fig. 7r-t). The gsk3 quadruple mutant showed a tendency to reduce procambial cells (Supplementary Fig. 8b), but did not show the formation of xylem cells adjacent to phloem cells (Fig. 7a,b). Then, we introduced SK11, SK12-RNAi into the $g s k 3$ quadruple mutant to produce a sextuple mutant for GSK3s. Since the gsk3 sextuple mutant was seedling lethal, we analysed the adjacency phenotype in $\mathrm{T} 1$ transgenic plants after confirming the reduced expression levels of SK11 and SK12 (Supplementary Fig. $7 \mathrm{~b}$ ). Five of nine independent lines exhibited the formation of xylem cells adjacent to phloem cells (Fig. $7 c, d$, and Supplementary Fig. 9). Consistently, bikinin, which inhibits completely the activity of all members of SKI and SKII and partly that of ATSK32 (ref. 33), caused a similar defect to that observed in the gsk3 sextuple mutant (Fig. 7c-e). The adjacency phenotype in $t d r$ results from the exhaustion of procambial cells by a combination of the inhibition of procambial cell proliferation and the consumption of procambial cells via enhanced xylem differentiation ${ }^{16,18}$. wox4-1 mutant, which shows only a defect in TDIF-dependent promotion of procambial cell proliferation in contrast to gsk3 mutants ${ }^{18,32}$, exhibits the reduction of procambial cell layers but not the adjacency of xylem and phloem cells ${ }^{18,32}$. Then, we investigated a genetic relationship between TDR-GSK3s and TDR-WOX4 pathways by using bikinin and wox4-1 mutant, and also using markers such as pAPL-GUS for phloem cells ${ }^{34}, p$ TDR-GUS for procambial cells ${ }^{16}$ and secondary wall thickenings for xylem cells. In the WT, $p A P L-G U S$ expression domain was spatially separated from xylem region by $p T D R-G U S$ expression domain, representing that several procambial cell layers exist between phloem and xylem cells (Fig. $7 \mathrm{f}-\mathrm{h})$. The treatment with bikinin occasionally $(<40 \%)$ caused the adjacency of pAPL-GUS expression domain and xylem region, and the loss of $p T D R-G U S$ expression domain between phloem and xylem cells (Fig. 7i-k). On the other hand, wox4-1 showed narrower gap region existing between PAPL-GUS expression domain and xylem region than that in the WT, but rarely did the adjacency of xylem and phloem cells, coinciding with previous reports ${ }^{18,32}$ (Fig. 7l-n). With the combination of wox4-1, bikinin induced xylem cells adjacent to phloem cells at a similar frequency $(<80 \%)($ Fig. $7 \mathrm{o}-\mathrm{q})$ to that in the $t d r-1$ (Fig. $7 \mathrm{r}-\mathrm{t}$ ), indicating an additive role of TDR-GSK3s and TDR-WOX4 pathways in the adjacency phenotype. The involvement of GSK3s in xylem differentiation was confirmed by a result that exogenous bikinin application induced differentiation of many xylem vessel cells ectopically in cotyledons (Fig. 8a,b,e-g). Collectively, these results suggest that TDR-GSK3s pathway contributes to the maintenance of procambial cells principally by inhibiting xylem cell differentiation.
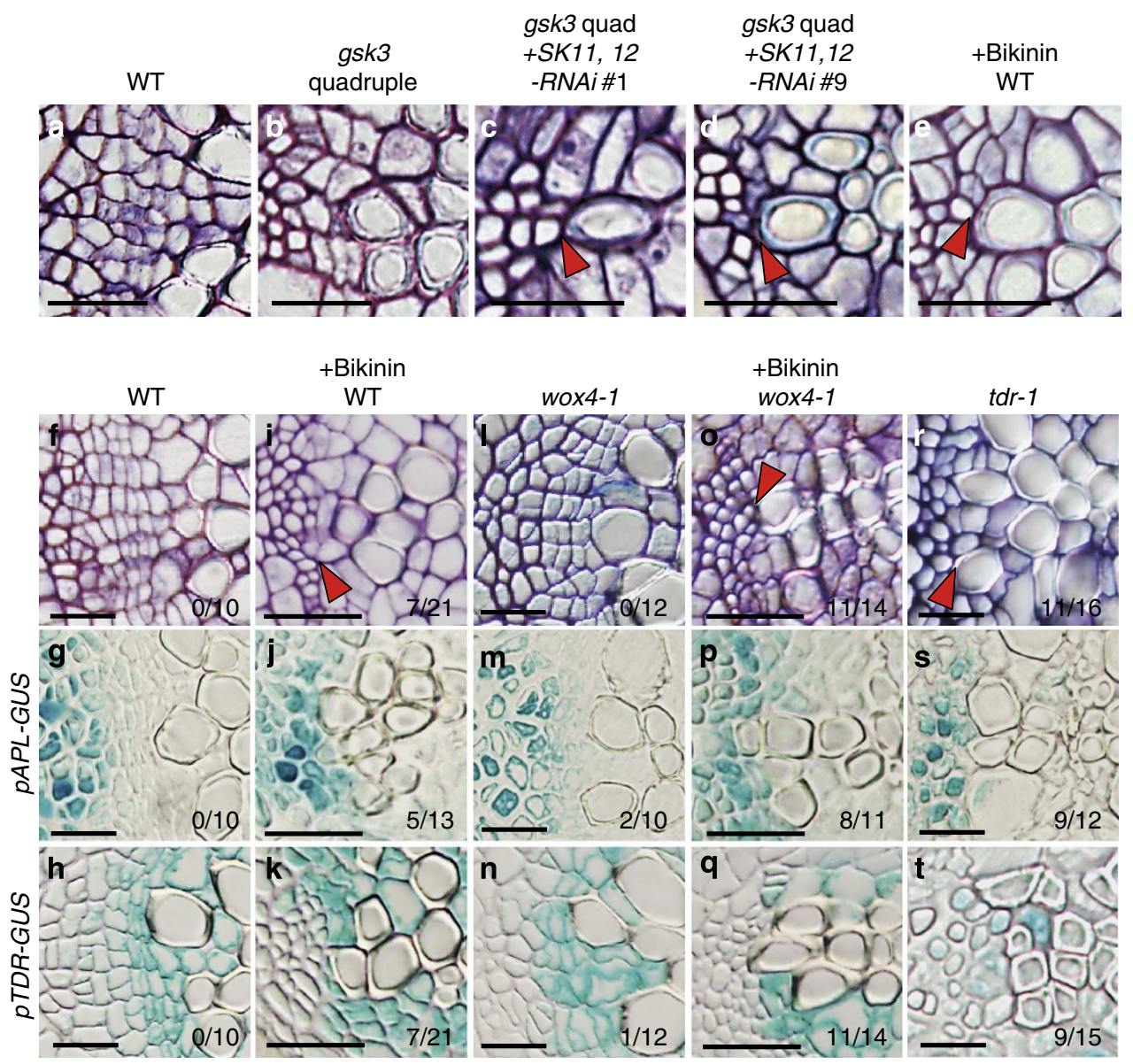

Figure 7 | Genetic analysis of GSK3s function in vascular development. (a-e) Cross sections of hypocotyls of seedlings grown for 11 days on MS agar plates with (e) or without bikinin (a-d); WT (a,e), gsk3 quadruple (b) and gsk3 sextuple mutants (c,d). (f-t) Cross sections of hypocotyls of seedlings grown for 11 days in MS liquid medium with (i-k, o-q) or without bikinin (f-h, I-n, r-t); WT (f,i), WT/pAPL-GUS (g,j), WT/pTDR-GUS (h,k),

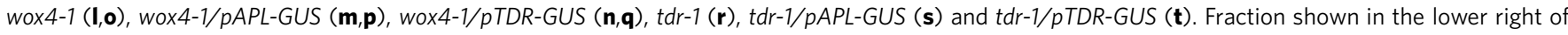
each picture indicates the number of plants that exhibited the adjacency of xylem and phloem cells per the total number of plants observed. Red arrowheads indicate the adjacency of xylem and phloem cells. Scale bars, $20 \mu \mathrm{m}$. 


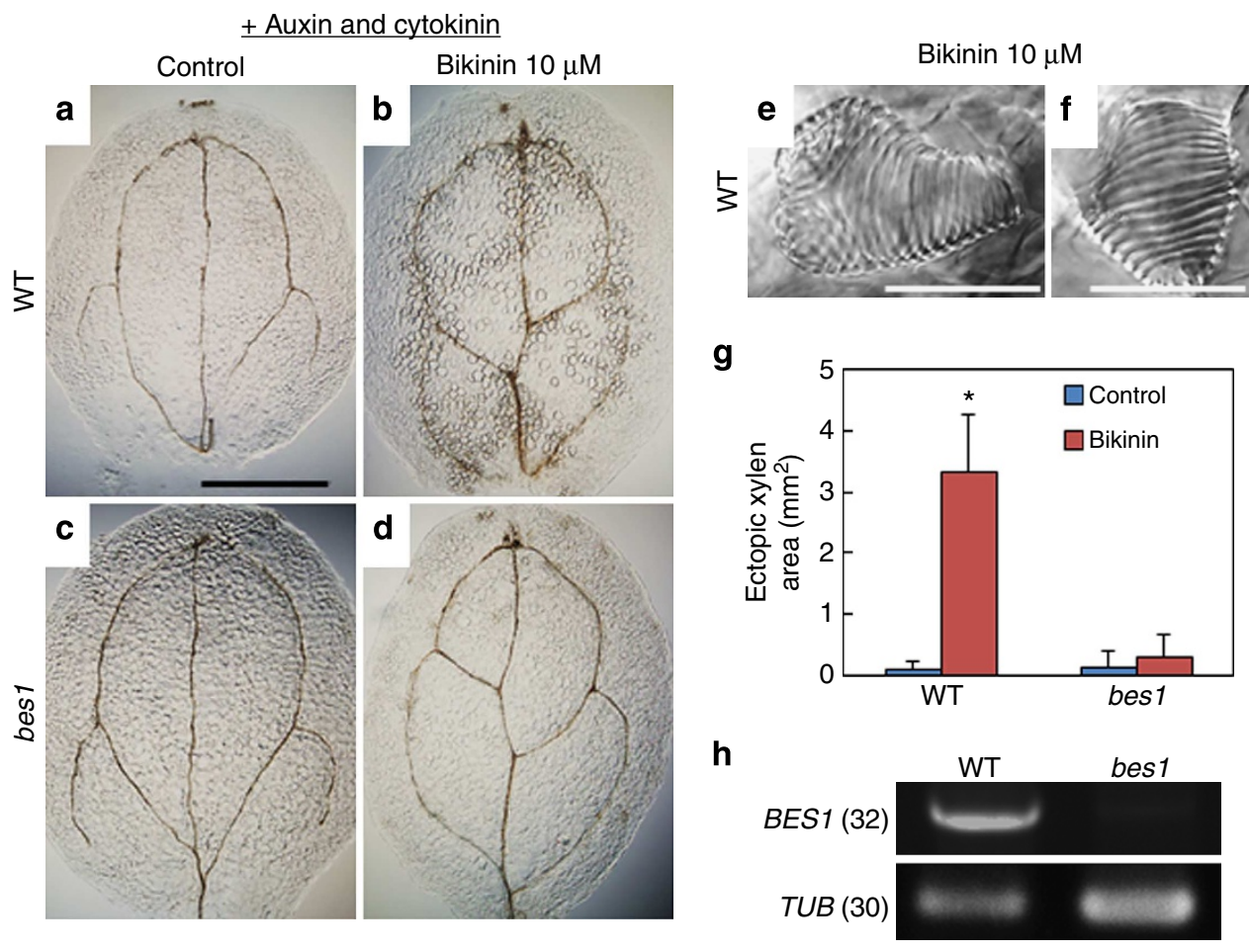

Figure 8 | BES1 is a target of GSK3s in the regulation of xylem differentiation. (a-d) Bikinin application induces ectopic induction of xylem vessel elements in cotyledons of WT but not of bes1, in the presence of auxin and cytokinin. (e,f) High magnification images of (b). (g) Ectopic xylem area was calculated in $(\mathbf{a}-\mathbf{d})(n=5)$. Error bar indicates s.d. Asterisks indicate significant differences using the Student's $t$-test $\left({ }^{\star} P<0.001\right)$. (h) $B E S 1$ expression levels in WT and bes1 were examined by semi-quantitative PCR with reverse transcription (RT-PCR). Beta-tubulin (TUB) was used as an internal control. Numbers written in right side indicate the amplification cycles in PCR. Scale bars, $1 \mathrm{~mm}$ for (a-d) and $50 \mu \mathrm{m}$ for (e,f).

BES1 is a main transcription factor in TDIF-GSK3s signalling. In BR signalling, GSK3s directly repress the activity of two similar transcription factors, BZR1 and BES1 (ref. 35). Next we examined the involvement of these transcription factors in xylem differentiation downstream of TDR-GSK3s. Of two dominant gain-of-function mutants ${ }^{28,36}$, bes1-D but not bzr1-D mutant reduced procambial cell layers and occasionally exhibited adjacency of xylem and phloem cells, resembling the phenotype of the gsk3 sextuple mutant (Fig. 9a,d,e). However, the adjacency phenotype of bes1-D is still weak, suggesting that other BES1-related transcription factors such as BES1/BZR1 HOMOLOGUEs (BEHs) may be involved in TDIF signalling ${ }^{37}$. Similarly to the case in bikinin-treated plants, the adjacency phenotype of bes1-D was enhanced by the wox4 mutant (Fig. 9e,f). In a loss-of-function mutant bes1 (Fig. 8h), bikinininduced ectopic xylem cell differentiation in cotyledons and adjacency of xylem and phloem cells in hypocotyls were barely observed (Figs 8a-g and $9 \mathrm{a}-\mathrm{c}$ ), indicating that BES1 is the main transcription factor that acts downstream of GSK3s.

\section{Discussion}

Here we demonstrate that GSK3/SHAGGY-like kinases including BIN2 redundantly play a crucial role in the regulation of xylem cell differentiation as downstream components of TDIF-TDR signalling. GSK3s have been implicated in multiple signalling pathways involved in developmental regulation in animals as well as in plants ${ }^{38}$. In mammals, there are only two isoforms of GSK3, alpha and beta, which play crucial roles in the various signal transduction pathways ${ }^{39,40}$. On the other hand, Arabidopsis GSK3s are encoded by ten genes ${ }^{38}$, consisting of four subgroups $^{22}$. Of them, SKI and II members redundantly mediate TDIF signalling, as suggested by $\mathrm{Y} 2 \mathrm{H}$, FRET and genetic analyses. Redundant roles of GSK3s have also been reported by studies on the BR signalling ${ }^{19,41}$, suggesting that functional redundancies among GSK3s generally contribute to the robustness in plant cell signalling.

GSK3s such as BIN2 are key regulators in the BR signalling as well as in the TDIF signalling. In the BR signalling at the cell surface, BRASSINOSTEROID-SIGNALLING KINASES (BSKs), which are known to function as cytoplasmic protein kinases, dissociate from BRI1 receptor complexes upon BRs perception ${ }^{42}$. This dissociation allows BSKs to interact with BRI1 SUPPRESSOR 1 (BSU1) ${ }^{42,43}$, which is a protein phosphatase and inactivates BIN2 by dephosphorylation ${ }^{43}$. We revealed that the PM-localized LRR-RLK TDR is closely associated with GSK3s including BIN2. In addition, TDIF application decreased FRET efficiency between TDR and BIN2, suggesting the dissociation of BIN2 from TDR or a change in spatial interrelationship between TDR and BIN2, which in turn may promote the access of BIN2 to its substrate BES1. Thus, in the two signalling pathways, ligand-dependent alteration of protein interaction at the PM initiates signal transduction similarly. However, there are major differences between the two signalling pathways. In the TDIF signalling, BIN2 activity is regulated directly by TDIF-TDR interaction, while in the BR signalling, BIN2 activity is not directly regulated by BR-BRI1 interaction but regulated through dephosphorylation by BSU1 (ref. 43). Furthermore, TDR activates GSK3s in a ligand-dependent manner in procambial cells, while GSK3s activity is controlled to be inactive by BSKs and BSU1 in the BR signalling ${ }^{43}$. Thus, our findings provide a novel insight into regulatory mechanism of GSK3s activity in ligand-receptor signalling pathways in plants.

Recently it has been reported that BIN2 connects the BR signalling with Mitogen-Activated Protein Kinase (MAPK) pathway in the regulation of stomatal development ${ }^{44,45}$. 

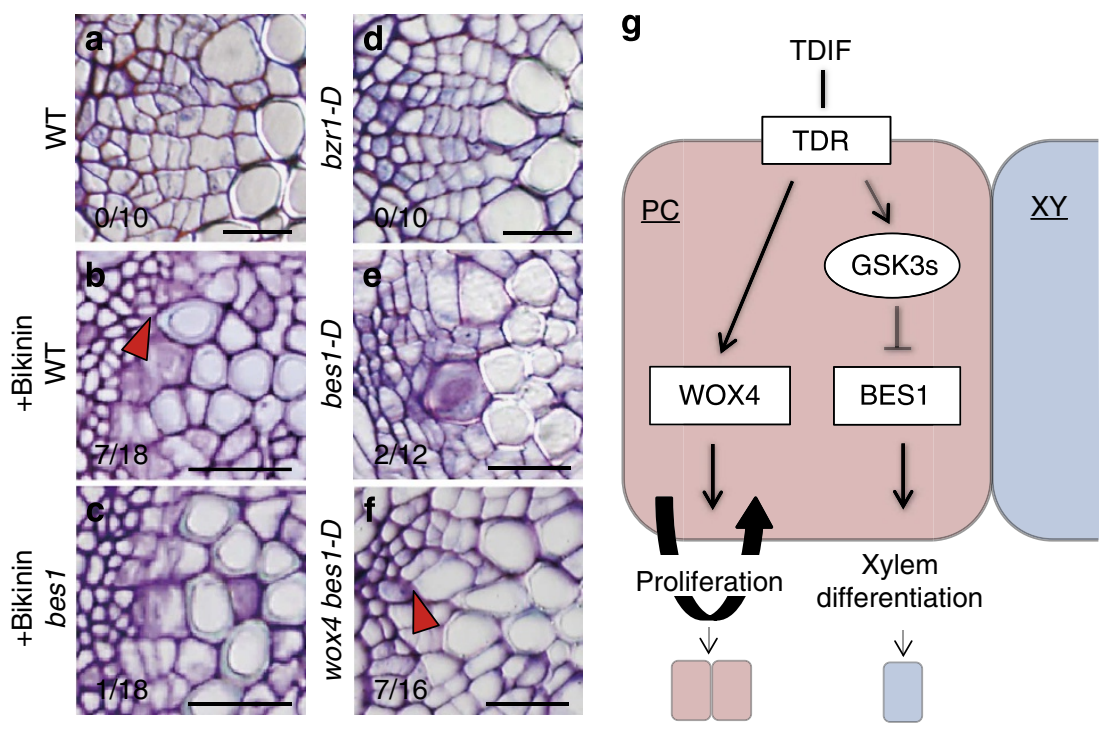

Figure 9 | Genetic relationship between TDR-BES1 and TDR-WOX4 pathways. (a-f) Cross sections of hypocotyls of seedlings grown for 11 days in MS liquid medium with $(\mathbf{b}, \mathbf{c})$ or without bikinin $(\mathbf{a}, \mathbf{d}-\mathbf{f})$; WT $(\mathbf{a}, \mathbf{b})$, bes1 (c), bzr1-D (d), bes1-D (e) and wox4 bes1-D (f). Red arrowheads indicate the adjacency of xylem and phloem cells. Fraction shown in the lower left of each picture indicates the number of plants that exhibited the adjacency of xylem and phloem cells per the total number of plants observed. (g) A model of TDIF-TDR signalling network in procambial cells governing both self-proliferation and differentiation into xylem cells. Scale bars, $20 \mu \mathrm{m}$ for (a-f).

BIN2 also functions as a hub between BR and auxin signalling pathways via phosphorylation of AUXIN RESPONSE FACTOR 2 (ARF2) by BIN2 (ref. 46). Therefore, plant GSK3s may function generally as critical hubs to connect the BR signalling pathway with other signalling pathways in various developmental processes. Indeed, because BRs promote xylem cell differentiation ${ }^{47,48}$, it will be interesting to analyse a cross-talk between TDIF and BR signalling through GSK3s in the regulation of xylem cell differentiation.

Genetic analysis revealed that BES1 acts as the main transcription factor in the TDIF-TDR-GSK3s signalling pathway. Since BES1 is a direct substrate of GSK3s in the BR signalling ${ }^{35}$, TDIF signalling pathway shares common signalling components with the BR signalling pathway. TDIF-TDR affected BZR1 localization via GSK3s activities, suggesting the possibility that TDIF signalling controls the activity of BZR1 as well as BES1. However, the gain-of-function mutant bzr1-D did not show obvious vascular phenotype, indicating a functional difference between BZR1 and BES1 probably due to distinct target genes ${ }^{35}$. Our genetic studies revealed that BES1 regulates the differentiation of procambial cells into xylem cells. Similarly, a previous study has demonstrated that BES1 is involved in the differentiation of columella stem cells ${ }^{49}$, suggesting a common role of BES1 in the regulation of stem cell differentiation.

Hirakawa et al. ${ }^{18}$ has presented a concept that TDIF signalling maintains procambial cells by suppressing their differentiation into xylem cells and promoting their proliferation via two distinct pathways ${ }^{5}$. In the regulation of cell proliferation, WUS and its family members play central roles ${ }^{50}$. Especially, WOX4 has been implicated in the promotion of cell proliferation in vascular meristems downstream of TDIF signalling ${ }^{18,32}$. By genetic analysis, we showed that GSK3s and BES1 function independently of WOX4 in the maintenance of procambial cells. Altogether, we illustrate a model for the maintenance of procambial cells, in which TDIF-TDR signalling is separated by two different pathways, TDR-GSK3s-BES1 and TDR-WOX4 pathway (Fig. 9g). In procambial cells, TDIF-TDR activates GSK3s via physical association, finally leading to the inactivation of the transcription factor BES1, which has an activity to promote xylem differentiation. As a consequence, xylem differentiation is suppressed by TDIF. On the other hand, TDIF-TDR increases the transcript of WOX4, which leads to the promotion of procambial cell proliferation ${ }^{16}$. Further analysis for finding target genes of transcription factors BES1 and WOX4 would provide an important insight into mechanisms regulating the differentiation and division of procambial cells.

\section{Methods}

Plant materials. bin2-3 and bin2-3billbil2 (Ws background) were provided by Dr Ildoo Hwang and Dr Jianming Li. bin2-3bill and bin2-3bil2 were made by crossing. gsk3 quadruple and sextuple mutants were generated from bin2-3bilibil2 with the use of RNAi. All other mutants in this study are in the Col-0 background. bzr1-D and bes1-D were gifts from Dr Takeshi Nakano. pTDR-GUS seeds (provided by Dr Taku Demura), $t d r-1$ and wox $4-1$ used in this study were reported previously ${ }^{16,18}$. Seeds of bes 1 mutant were received from the ABRC stock centre (stock ID, SALK_091133C).

Yeast two-hybrid assay. For the Y2H screening, DNA sequence encoding the TDR intracellular domain was cloned into pGBKT7 vector and then was transformed to the Y187 yeast strain as bait. A prey library was constructed from Arabidopsis root cDNA using Matchmaker Two-Hybrid Library Construction \& Screening Kit (Clontech) and was introduced into the AH109 strain. Screening was performed according to the mating protocol (Clontech). Selection was performed for 5 days on SD plates without tryptophan, leucine and histidine at $27^{\circ} \mathrm{C}$. Insertion sequences of positive clones were amplified and sequenced to identify the corresponding genes. For the confirmation of interaction, BIN2, BIL1, BIL2, SK11, SK12, SK13, SK31, SK32, SK41, SK42, cytoplasmic regions of TDR and CRN were cloned into pACTII and pGBKT7 and subjected to further $\mathrm{Y} 2 \mathrm{H}$ assay. AH109 yeast strains, into which pACTII and pGBKT7 are co-transformed, were cultured until the optical density (OD) reached 0.4 and then $1 / 10$ dilutions were spotted on SD plates without tryptophan, leucine or histidine. Plates were incubated for 2 days at $27^{\circ} \mathrm{C}$. Y2H spot analysis was performed with two different yeast colonies and was repeated with two independent sets.

$\mathbf{N}$. benthamiana transient assay and FRET analysis. Rhizobium radiobacter strain GV3101 MP90 carrying expression constructs was incubated with LB liquid medium (MERCK) for 1 day with shaking and then centrifuged at $1,800 \mathrm{~g}$ for $10 \mathrm{~min}$. Pellets were resuspended in infiltration buffer $(10 \mathrm{mM} \mathrm{MES}, 10 \mathrm{mM} \mathrm{MgCl}$ and $150 \mu \mathrm{M}$ acetosyringone; $\mathrm{pH}$ 5.7). After the optical density was adjusted to 1.0, each buffer containing agrobacterium was mixed with the same volume of cultures harbouring a p19k suppressor construct ${ }^{51}$. The mixed cultures were injected into $N$. benthamiana leaves with $1 \mathrm{ml}$ syringe (Terumo). An estradiol-inducible system was used to express TDR-CFP, because the CaMV 35 S promoter-driven TDR-YFP 
was not stably expressed (Supplementary Fig. 4a,b). Estradiol induction was performed by incubating leaf disks with $10 \mu \mathrm{M}$ estradiol for 6-24h. For the observation of fluorescent signals, injected and estradiol-treated leaves were cut off and degassed for $5 \mathrm{~min}$ to eliminate auto-fluorescence at the cell surface. Fluorescent signal was detected with a fluorescent microscope BX51 (Olympus). For the protein localization analysis, transient assay was repeated at least three times. Intactness of introduced proteins was confirmed by the facts that TDR-GFP complemented the $t d r$ mutant phenotype and that degradation of TDR-CFP as well as BIN2-YFP was not detected (Supplementary Fig. 10). FRET analysis was performed according to the acceptor photobleaching method using a confocal microscope LSM-510 META (Carl Zeiss). Photobleach was done by $514 \mathrm{~nm}$ laser irradiation, repeated 50 times. The excitation wavelength was $458 \mathrm{~nm}$ and $514 \mathrm{~nm}$ for detecting fluorescent signal of CFP and YFP, respectively. These emissions were collected at $477-520 \mathrm{~nm}$ and $531-563 \mathrm{~nm}$ for CFP and YFP, respectively. FRET efficiency was calculated according to the equation; FRET efficiency $(\%)=((\mathrm{Ca}-\mathrm{Ba})$ $-(\mathrm{Cb}-\mathrm{Bb})) /(\mathrm{Ca}-\mathrm{Ba}) \times 100$. Ca; CFP signal after photobleaching. Ba: background signal after photobleaching. Cb: CFP signal before photobleaching. Bb: background signal before photobleaching. Used primers were listed in Supplementary Table 2.

Detection of BZR1-GFP movement in Arabidopsis. Five-day-old seedlings of transgenic plants carrying the $p T D R-B Z R 1-s G F P$ vector were treated with TDIF or bikinin for $1 \mathrm{~h}$ and then small true leaves were isolated from seedlings under a stereomicroscope. Fluorescent signal was observed under an inverted fluorescent microscope (IX70; Olympus) equipped with a confocal unit (CSU10; Yokogawa). The ratios of fluorescent signal intensity in the cytosol and nucleus were calculated by analysing obtained images using Image J (http://rsbweb.nih.gov/ij/). pTDRBZR1-sGFP vector was constructed with R4pGWB504 (from Dr Nakagawa) by using the MultiSite Gateway system (Invitrogen), and introduced into the WT and the $t d r-1$. Used primers were listed in Supplementary Table 2.

Xylem cell induction by bikinin in Arabidopsis cotyledons. Six-day-old Arabidopsis seedlings were transferred to MS-based liquid medium (2.2 g MS; $50 \mathrm{~g}$ glucose per litre, $\mathrm{pH}$ 5.7) containing $1.25 \mathrm{mgl}^{-1}$ 2,4-dichlorophenoxyacetic acid, $0.25 \mathrm{mgl}^{-1}$ kinetin and $10 \mu \mathrm{M}$ bikinin (ChemBridge, USA) and then cultured for 3 days in 12 multi-well plates (Sumilon, Japan). Culture conditions are at $22^{\circ} \mathrm{C}$ under continuous light with shaking at 120 r.p.m. For quantification, areas for ectopic xylem cells in the cotyledons were calculated using Image J.

Pull-down analysis. An E.coli strain BL21 (Invitrogen) carrying plasmids for expression of GST-GFP, GST-BIN2, GST-BIL1 and 6His-TDRKD was cultured at $16^{\circ} \mathrm{C}$ for 1 day with $20 \% \mathrm{~L}$-arabinose (Sigma). Total proteins were extracted from BL21 culture and dissolved with extraction buffer $(100 \mathrm{mM}$ Tris- $\mathrm{HCl}$ pH8.0, $150 \mathrm{mM} \mathrm{NaCl}$, and proteinase inhibitor cocktail (Sigma)) and $1 \mathrm{mg} \mathrm{ml}^{-1}$ lysozyme (Wako). For purification, total protein including 6His-TDRKD was captured by Ni-NTA agarose column (Qiagen) and then washed with extraction buffer three times. Purified 6His-TDRKD was eluted with extraction buffer containing $200 \mathrm{mM}$ imidazole (Wako). For pull-down assay, total protein from BL21 expressing GSTGFP, GST-BIN2 or GST-BIL1 was mixed with purified 6His-TDRKD. After 1-hour incubation, samples were applied onto glutathione Sepharose 4B columns (GE Healthcare). Columns were washed with extraction buffer three times and then eluted with extraction buffer containing $10 \mathrm{mM}$ reduced glutathione (Wako). Obtained protein was analysed by CBB staining (Wako) and western blotting with anti-6His-HRP antibody (1:1,000, Qiagen; catalogue number 34460) after SDS-PAGE. Pull-down analysis was repeated with two independent sets.

Observation of vasculature. For the observation, samples were fixed with a mixture of acetic acid/ethanol (1:3) and transferred into the clearing solution (chloral hydrate/glycerol/water 8:1:2). For the cross sections, hypocotyls of 11-dayold seedlings were fixed with FAA (Formalin/Acetic acid/Alcohol; 1:1:18) for 1 day and then transferred to ethanol series $(50 \%, 70 \%, 80 \%, 90 \%$ and $99.5 \%$; in this order) for 15-30 min. After that, fixed hypocotyls were embedded in single PCR tubes with Technovit 7100 (Kulzer). Embedded samples were sliced at an interval of $4 \mu \mathrm{m}$ thickness by LEICA RM2165 (Leica). Sliced samples were stained with $0.1 \%$ toluidine blue for $1 \mathrm{~min}$. Vascular cell number was counted from hypocotyl transverse section images of 12 independent individuals in the WT and mutants.

GUS staining. Tissues were fixed in $90 \%$ acetone for $1 \mathrm{~h}$ at $-20^{\circ} \mathrm{C}$, and washed twice with sodium phosphate buffer ( $\mathrm{pH} 7.0)$. Washed samples were immersed in GUS staining solution ( $100 \mathrm{mM}$ sodium phosphate buffer, $10 \mathrm{mM}$ EDTA, $0.1 \%$ Triton X-100, $1 \mathrm{mg} \mathrm{ml}^{-1}$ 5-bromo-4-chloro-3-indolyl b-D-glucuronide [X-gluc], $2.5 \mathrm{mM}$ potassium ferricyanide and $2.5 \mathrm{mM}$ potassium ferrocyanide) for $1-4 \mathrm{~h}(\mathrm{~s})$ at $37^{\circ} \mathrm{C}$. Reactions were stopped in $70 \%$ ethanol.

Knockdown for SK11, SK12 and SK13. The RNAi construct containing the last $350 \mathrm{bp}$ of SK13 cDNA including $3^{\prime}$ UTR region and intron of WRKY33 as a linker was driven under $35 \mathrm{~S}$ promoter and introduced into the bin 2 triple mutant. We obtained three independent T3 lines and used the severest line T3\#4. Similarly, we made an RNAi construct against SK11 and SK12 using the most conserved region of coding sequences among SK11 and SK12. Sextuple mutants were obtained by introducing $S K 11,12-R N A i$ into the gsk3 quadruple T3\#4 for further analysis. Vascular phenotype was analysed in T1 generation because of their seedling-lethal phenotypes. At the same time, we confirmed the downregulation of SK11 and SK12 by quantitative real-time PCR against T1 individuals.

Promoter-GUS analysis. To construct $p B I N 2-G U S, p B I L 1-G U S$ and $p B I L 2-G U S$ vector, we cloned an approximately $1-2 \mathrm{~kb}$ DNA fragment upstream of the predicted start codon of each gene and introduced it into pMDC163. GUS staining was observed in no less than five independent lines for pBIN2-GUS, pBIL1-GUS and pBIL2-GUS transgenic plants. Used primers were listed in Supplementary Table 2.

Quantitative PCR with reverse transcription (RT-PCR). Total RNA was extracted from seedlings using RNeasy plant mini kit (Qiagen). Quantitative PCR was performed using Light Cycler (Roche Diagnostics). For the calculation of relative expression, TUA4 was used as an internal control for normalization. Gene-specific primer sets are listed in Supplementary Table 2.

Western blotting. Total protein was extracted from infiltrated $N$. benthamiana leaf disks, according to the previous method ${ }^{10}$. anti-GFP (1:1000, Roche; catalogue number 11814460001) and anti-GFP-HRP (1:1000, Miltenyi Biotec; catalogue number 130-091-833) were used for detecting CFP and YFP proteins. For the expression analysis in yeasts, total protein was extracted from yeast culture and analysed with anti-c-Myc antibody 9E10 (1:5000, Roche; catalogue number 11667149001). Western blotting analyses were repeated with two independent sets.

Complementation analysis. $p T D R-T D R-G 3 G F P$ vector was constructed with pGWB551 (from Dr Nakagawa) by using the MultiSite Gateway system (Invitrogen), and transformed into the $t d r-1$ mutant. T2 seeds (independent eigh lines) were grown for 10 days in liquid MS medium containing $1000 \mathrm{nM}$ TDIF.

\section{References}

1. Perales, M. \& Reddy, G. V. Stem cell maintenance in shoot apical meristems. Curr. Opin. Plant Biol. 15, 10-16 (2012).

2. Aichinger, E., Kornet, N., Friedrich, T. \& Laux, T. Plant stem cell niches. Annu. Rev. Plant Biol. 63, 615-636 (2012).

3. Stahl, Y. \& Simon, R. Plant stem cell niches. Int. J. Dev. Biol. 49, 479-489 (2005).

4. Fletcher, J. C. Signaling of cell fate decisions by CLAVATA3 in Arabidopsis shoot meristems. Science 283, 1911-1914 (1999).

5. Clark, S. E., Williams, R. W. \& Meyerowitz, E. M. The CLAVATA1 gene encodes a putative receptor kinase that controls shoot and floral meristem size in Arabidopsis. Cell 89, 575-585 (1997).

6. Mayer, K. F. X. et al. Role of WUSCHEL in regulating stem cell fate in the Arabidopsis shoot meristem. Cell 95, 805-815 (1998).

7. Fiers, M. et al. The CLAVATA3/ESR motif of CLAVATA3 is functionally independent from the nonconserved flanking sequences. Plant Physiol. 141, 1284-1292 (2006).

8. Ogawa, M., Shinohara, H., Sakagami, Y. \& Matsubayashi, Y. Arabidopsis CLV3 peptide directly binds CLV1 ectodomain. Science 319, 294 (2008).

9. Muller, R., Bleckmann, A. \& Simon, R. The receptor kinase CORYNE of Arabidopsis transmits the stem cell-limiting signal CLAVATA3 independently of CLAVATA1. Plant Cell 20, 934-946 (2008).

10. Kinoshita, A. et al. RPK2 is an essential receptor-like kinase that transmits the CLV3 signal in Arabidopsis. Development 137, 3911-3920 (2010).

11. Brand, U., Fletcher, J. C., Hobe, M., Meyerowitz, E. M. \& Simon, R. Dependence of stem cell fate in Arabidopsis on a feedback loop regulated by CLV3 activity. Science 289, 617-619 (2000)

12. Schoof, H. et al. The stem cell population of Arabidopsis shoot meristems is maintained by a regulatory loop between the CLAVATA and WUSCHEL genes. Cell 100, 635-644 (2000).

13. Yadav, R. K. et al. WUSCHEL protein movement mediates stem cell homeostasis in the Arabidopsis shoot apex. Genes Dev. 25, 2025-2030 (2011).

14. Stahl, Y., Wink, R. H., Ingram, G. C. \& Simon, R. A signaling module controlling the stem cell niche in Arabidopsis root meristems. Curr. Biol. 19, 909-914 (2009).

15. Ito, Y. et al. Dodeca-CLE peptides as suppressors of plant stem cell differentiation. Science 313, 842-845 (2006).

16. Hirakawa, Y. et al. Non-cell-autonomous control of vascular stem cell fate by a CLE peptide/receptor system. Proc. Natl Acad. Sci. USA 105, 15208-15213 (2008).

17. Fisher, K. \& Turner, S. PXY, a receptor-like kinase essential for maintaining polarity during plant vascular-tissue development. Curr. Biol. 17, 1061-1066 (2007). 
18. Hirakawa, Y., Kondo, Y. \& Fukuda, H. TDIF peptide signaling regulates vascular stem cell proliferation via the WOX4 homeobox gene in Arabidopsis. Plant Cell 22, 2618-2629 (2010).

19. Li, J. \& Nam, K. H. Regulation of brassinosteroid signaling by a GSK3/ SHAGGY-like kinase. Science 295, 1299-1301 (2002).

20. Miwa, H. et al. The receptor-like kinase SOL2 mediates CLE signaling in Arabidopsis. Plant Cell Physiol. 49, 1752-1757 (2008).

21. Kondo, Y., Hirakawa, Y., Kieber, J. J. \& Fukuda, H. CLE peptides can negatively regulate protoxylem vessel formation via cytokinin signaling. Plant Cell Physiol. 52, 37-48 (2011).

22. Saidi, Y., Hearn, T. J. \& Coates, J. C. Function and evolution of 'green' GSK3/Shaggy-like kinases. Trends Plant Sci. 17, 39-46 (2012).

23. Vert, G. \& Chory, J. Downstream nuclear events in brassinosteroid signalling. Nature 441, 96-100 (2006).

24. Bleckmann, A., Weidtkamp-Peters, S., Seidel, C. A. \& Simon, R. Stem cell signaling in Arabidopsis requires CRN to localize CLV2 to the plasma membrane. Plant Physiol. 152, 166-176 (2010).

25. Miyawaki, A. \& Tsien, R. Y. Monitoring protein conformations and interactions by fluorescence resonance energy transfer between mutants of green fluorescent protein. Method Enzymol 327, 472-500 (2000).

26. Stone, J. M., Trotochaud, A. E., Walker, J. C. \& Clark, S. E. Control of meristem development by CLAVATA1 receptor kinase and kinase-associated protein phosphatase interactions. Plant Physiol. 117, 1217-1225 (1998).

27. Ryu, H. et al. Nucleocytoplasmic shuttling of BZR1 mediated by phosphorylation is essential in Arabidopsis brassinosteroid signaling. Plant Cell 19, 2749-2762 (2007).

28. Wang, Z. Y. et al. Nuclear-localized BZR1 mediates brassinosteroid-induced growth and feedback suppression of brassinosteroid biosynthesis. Dev. Cell 2, 505-513 (2002)

29. Ryu, H., Cho, H., Kim, K. \& Hwang, I. Phosphorylation dependent nucleocytoplasmic shuttling of BES1 is a key regulatory event in brassinosteroid signaling. Mol. Cells 29, 283-290 (2010).

30. Gampala, S. S. et al. An essential role for 14-3-3 proteins in brassinosteroid signal transduction in Arabidopsis. Dev. Cell 13, 177-189 (2007).

31. Etchells, J. P. \& Turner, S. R. The PXY-CLE41 receptor ligand pair defines a multifunctional pathway that controls the rate and orientation of vascular cell division. Development 137, 767-774 (2010).

32. Etchells, J. P., Provost, C. M., Mishra, L. \& Turner, S. R. WOX4 and WOX14 act downstream of the PXY receptor kinase to regulate plant vascular proliferation independently of any role in vascular organisation. Development 140, 2224-2234 (2013).

33. De Rybel, B. et al. Chemical inhibition of a subset of Arabidopsis thaliana GSK3-like kinases activates brassinosteroid signaling. Chem. Biol. 16, 594-604 (2009).

34. Bonke, M., Thitamadee, S., Mahonen, A. P., Hauser, M. T. \& Helariutta, Y. APL regulates vascular tissue identity in Arabidopsis. Nature 426, 181-186 (2003).

35. Wang, Z. Y., Bai, M. Y., Oh, E. \& Zhu, J. Y. Brassinosteroid signaling network and regulation of photomorphogenesis. Annu. Rev. Genet. 46, 701-724 (2012).

36. Yin, Y. H. et al. BES1 accumulates in the nucleus in response to brassinosteroids to regulate gene expression and promote stem elongation. Cell 109, 181-191 (2002).

37. Yin, Y. et al. A new class of transcription factors mediates brassinosteroidregulated gene expression in Arabidopsis. Cell 120, 249-259 (2005).

38. Jonak, C. \& Hirt, H. Glycogen synthase kinase 3/SHAGGY-like kinases in plants: an emerging family with novel functions. Trends Plant Sci. 7, 457-461 (2002).

39. Forde, J. E. \& Dale, T. C. Glycogen synthase kinase 3: a key regulator of cellular fate. Cell. Mol. Life Sci. 64, 1930-1944 (2007).
40. Hur, E. M. \& Zhou, F. Q. GSK3 signalling in neural development. Nat. Rev. Neurosci. 11, 539-551 (2010).

41. Yan, Z., Zhao, J., Peng, P., Chihara, R. K. \& Li, J. BIN2 functions redundantly with other Arabidopsis GSK3-like kinases to regulate brassinosteroid signaling. Plant Physiol. 150, 710-721 (2009).

42. Tang, W. et al. BSKs mediate signal transduction from the receptor kinase BRI1 in Arabidopsis. Science 321, 557-560 (2008).

43. Kim, T. W. et al. Brassinosteroid signal transduction from cell-surface receptor kinases to nuclear transcription factors. Nat. Cell Biol. 11, 1254-1260 (2009).

44. Gudesblat, G. E. et al. SPEECHLESS integrates brassinosteroid and stomata signalling pathways. Nat. Cell Biol. 14, 548-554 (2012).

45. Kim, T. W., Michniewicz, M., Bergmann, D. C. \& Wang, Z. Y. Brassinosteroid regulates stomatal development by GSK3-mediated inhibition of a MAPK pathway. Nature 482, 419-422 (2012).

46. Vert, G., Walcher, C. L., Chory, J. \& Nemhauser, J. L. Integration of auxin and brassinosteroid pathways by Auxin Response Factor 2. Proc. Natl Acad. Sci. USA 105, 9829-9834 (2008).

47. Yamamoto, R. et al. Co-regulation of brassinosteroid biosynthesis-related genes during xylem cell differentiation. Plant Cell Physiol. 48, 74-83 (2007).

48. Cano-Delgado, A. et al. BRL1 and BRL3 are novel brassinosteroid receptors that function in vascular differentiation in Arabidopsis. Development 131, 5341-5351 (2004).

49. Gonzalez-Garcia, M. P. et al. Brassinosteroids control meristem size by promoting cell cycle progression in Arabidopsis roots. Development 138, 849-859 (2011).

50. van der Graaff, E., Laux, T. \& Rensing, S. A. The WUS homeobox-containing (WOX) protein family. Genome Biol. 10, 248 (2009).

51. Voinnet, O., Rivas, S., Mestre, P. \& Baulcombe, D. An enhanced transient expression system in plants based on suppression of gene silencing by the p19 protein of tomato bushy stunt virus. Plant J. 33, 949-956 (2003).

\section{Acknowledgements}

We thank Kazuo Ebine, Takashi Ueda, Ichiro Terashima, Shigeyuki Betsuyaku, Kuninori Iwamoto and Yasuko Ozawa for technical supports; and Ildoo Hwang, Jianming Li, Takeshi Nakano and Taku Demura for providing plant materials. This work was supported partly by Grants-in-Aid from the Ministry of Education, Science, Sports and Culture of Japan (19060009, NC-CARP project) to HF, from the Japan Society for the Promotion of Science (23227001 to HF, 24228008 to KS, 24688007 to HN and JSPS Research Fellowships for Young Scientists to YK), a long-term fellowship from the Human Frontier Science Program to YH, and from Bio-oriented Technology Research Advancement Institution (BRAIN) to HF.

\section{Author contributions}

Y.K., T.I., H.N., Y.H., M.S., T.T. and K.S. performed the genetic, biochemical and physiological analysis of TDR and GSK3s. Y.K. and H.F. wrote the paper.

\section{Additional information}

Supplementary Information accompanies this paper at http://www.nature.com/nature communications

Competing financial interests: The authors declare no competing financial interests.

Reprints and permission information is available online at http://npg.nature.com/ reprintsandpermissions/

How to cite this article: Kondo, Y. et al. Plant GSK3 proteins regulate xylem cell differentiation downstream of TDIF-TDR signalling. Nat. Commun. 5:3504 doi: $10.1038 /$ ncomms4504 (2014). 